Voix et Images

voixetimages

\title{
Embrayage et modalisation dans l'incipit de la fiction fantastique brève
}

\section{André Carpentier}

Volume 24, numéro 1 (70), automne 1998

Yves Préfontaine

URI : https://id.erudit.org/iderudit/201411ar

DOI : https://doi.org/10.7202/201411ar

Aller au sommaire du numéro

\section{Éditeur(s)}

Université du Québec à Montréal

\section{ISSN}

0318-9201 (imprimé)

1705-933X (numérique)

Découvrir la revue

Citer cet article

Carpentier, A. (1998). Embrayage et modalisation dans l'incipit de la fiction fantastique brève. Voix et Images, 24(1), 141-150.

https://doi.org/10.7202/201411ar
Résumé de l'article

Ce texte porte sur une double caractéristique propre à l'ouverture de certains textes fantastiques, soit les motifs de rationalité et les motifs du surnaturel. À partir des premières pages de la nouvelle « Les cyclopes du jardin public » de Marie José Thériault et s'appuyant sur une conception du fantastique inspirée de Roger Caillois et de Louis Vax, ainsi que des travaux de Charles Grivel, l'analyse portera sur ce qu'il est convenu d'appeler l'incipit, au sens non restrictif de l'ouverture du texte fantastique. 


\title{
Embrayage et modalisation dans l'incipit de la fiction fantastique brève
}

\author{
André Carpentier, Université du Québec à Montréal
}

Ce texte porte sur une double caractéristique propre à l'ouverture de certains textes fantastiques, soit les motifs de rationalité et les motifs du surnaturel. À partir des premières pages de la nouvelle "Les cyclopes du jardin public" de Marie José Thériault et s'appuyant sur une conception du fantastique inspirée de Roger Caillois et de Louis Vax, ainsi que des travaux de Charles Grivel, l'analyse portera sur ce qu'il est convenu d'appeler. l'incipit, au sens non restrictif de l'ouverture du texte fantastique.

L'inquiétude fantastique, comme toute inquiétude, se nourrit de doutes plutôt que de certitude. Un récit [...] a besoin, comme un chagrin d'amour, d'alternances d'espoir et de détresse. [...]

[Le récit fantastique] a l'air de vouloir cacher quelque chose parce qu'il veut de nous cet état d'incertitude, parce qu'il n'a jamais été qu'une incertitude parfaitement délimitée, et qui se donne pour telle. [...]

C'est une incertitude tout à la fois diffuse et précise qui perce sous les allusions, les paroles ambiguës [...]

Louis Vax, La séduction de l'étrange ${ }^{1}$

J'aborderai ici, dans un mouvement spiralé autour de la question, une double caractéristique propre à l'ouverture de certains textes fantastiques. Cette particularité se condense en des formules de fantasticité, soit insinuantes, soit atténuantes, oscillant entre des motifs de rationalité et des motifs de surnaturel (ou d'étrangeté).

1. Louis Vax, La séduction de l'étrange, Paris, Presses universitaires de France, coll. "Quadrige $n$, 1965, p. 129-130. 
Ces motifs ont non seulement pour but d'exprimer la dichotomie rationalité/surnaturel, mais visent également à éviter la confusion entre le surnaturel comme unité figurative "traduct[rice] du monde surnaturel ${ }^{2}$, et le fantastique lui-même, comme genre du discours ${ }^{3}$. Les motifs du surnaturel consistent en des variables autonomes, parallèles aux articulations narratives, et apparaissent dans le texte fantastique en tant qu'éléments constituants. Quant aux motifs de rationalité, ils renvoient à des figures contribuant à la représentation du monde avéré et de la raison commune - autres constituants du type de fantastique qui m'intéresse ici.

Je commencerai en m'appuyant sur une proposition de l'essayiste français Roger Caillois: "Le fantastique [...] manifeste un scandale, une déchirure, une irruption insolite, presque insupportable dans le monde réel ${ }^{4}$. Scandale, du grec skandalon, "obstacle", emprunté à l'hébreu mikchôl, "qui fait trébucher", et employé au sens figuré dans les Évangiles pour signifier une occasion de chute fournie par un mauvais exemple. J'emploierai donc le mot "scandale" au sens de ce qui fait trébucher la raison, car je veux justement comprendre le texte fantastique comme une manifestation du scandale de la déraison, qui se produit lorsque la représentation du surnaturel vient mettre en péril (la représentation de) la raison commune. Mon objectif est d'illustrer la stratégie rhétorique qu'utilise le fantastique (ou plutôt un certain fantastique) pour induire cette déraison.

Il y aurait donc scandale lorsque la raison commune et la démarche cognitive habituelle cessent de dominer la perception. La présence de hiatus dans la rationalité, plus ou moins dicibles, souvent quasi innommables (étymologiquement: des béances, des déchirures, disait Caillois), et les effets inférés par ces hiatus apparaissent scandaleux, comme paraît choquant tout ce qui, faisant irruption dans l'ordre rationnel, y résiste et transgresse la logique collective et cohésive. Il y a scandale, évidemment, parce qu'est alors perverti le cadre des idées reçues - idées reçues qui, soit dit en passant, assurent moins qu'elles ne définissent l'idéal d'un équilibre rationnel. Le fantastique est donc désordre et différence, qui rompt la vision intégrée du monde, si rassurante, si rassembleuse! La raison craint ce moment de défaillance, ce délire du fantastique, qui engage une déformation consentie de la rationalité.

En d'autres termes, c'est moins la déraison elle-même que la rupture de raison qui semble traumatisante. Le merveilleux, par exemple, forme

2. Michel Guiomar, Principes d'une esthétique de la mort, Paris, José Corti, 1988, p. 277.

3. Sur cette question du surnaturel et du fantastique, voir les nuances apportées par Michel Lord, La logique de l'impossible. Aspects du discours fantastique québécois, Québec, Nuit blanche éditeur, 1995; p. 42-43.

4. Roger Caillois, "Images, images... ", Obliques précédé de Images, images..., Paris, Gallimard, 1987, p. 18. 
homogène de la déraison, fait moins trébucher la raison que le fantastique, genre hétérogène, qui présente des parties dont le dessein diffère, généralement par simulation de perte d'unité rationnelle et relationnelle du système textuel - c'est ce que nous verrons plus loin. En ce sens, le principe de "distanciation cognitive" que Darko Suvin applique à la science-fiction - qui se penche sur le prolongement, projectif et spéculatif, d'un monde ${ }^{5}$, soit-il anxiogène - serait contraire à la tentation de l'étrange propre au fantastique. La disposition mentale de la sciencefiction est celle de la conjecture rationnelle - au sens d'une opinion fondée sur des probabilités; celle du fantastique renvoie au principe d'une conjecture irrationnelle, comprise ici au sens d'une opinion fondée sur des apparences, donc comme une perception, une perception qui s'infiltre dans les failles de la rationalité pour en brouiller les certitudes, en se donnant comme objet l'étrange ou le surnaturel.

Ce traumatisme constitutif du fantastique auquel je fais référence, traumatisme face à la perspective de l'hétérogène, ou plutôt face à l'hétérogénéité du système, renverrait à une angoisse de déconstruction, dont on reconnaît la manifestation dans le texte fantastique sous des formes hybrides de morcellement, d'écroulement, de démembrement, de décomposition, etc., toutes formes de déconstruction, donc, qui agitent les spectres d'une dislocation de soi et de la fin d'un monde.

Dans le fantastique, ce que j'ai réduit aux motifs du surnaturel empiète sur les motifs de la rationalité ${ }^{6}$, parfois même en renverse les effets. Il y a là deux poursuites de sens qui s'opposent, deux quêtes irréconciliables, sauf à vouloir se rendre étranger à soi-même. Or, c'est là, justement, que se joue cette "séduction de l'étrange", dont parle Louis Vax, dans la tentation jouissive de frôler le danger de déconstruction: "La peur, pour persévérer, se cherche des raisons d'avoir peur ${ }^{7}$. "Et le même de dire ailleurs: "[...] dans le fantastique: le mal reste mauvais, la laideur atroce. Simplement, il y a complaisance pour l'un et pour l'autre. Un plaisir positif est cherché dans l'expérience d'un sentiment négatif ${ }^{8}$." Il y aurait donc jouissance esthétique (ou jouissance fantastique), ou encore "plaisir de l'indétermination ", comme le suggère Rachel Bouvet, à "se laisser happer par le vide, [à] ne rien offrir en compensation, [à] se laisser

5. Darko Suvin, Pour une poétique de la science-fiction, Montréal, Presses de l'Université dú Québec, coll. "Genres et discours", 1977, p. 15: "La science-fiction est donc un genre littéraire dont les conditions nécessaires et suffisantes sont la présence et l'interaction de la distanciation et de la connaissance, et dont le principal procédé formel est un cadre imaginaire, différent du monde empirique de l'auteur, "

6. La rationalité, non comme processus discursif, mais comme forme de thématisation de la raison commune, donc comme procédure textuelle prenant en charge les valeurs de représentation d'un ordre rationnel.

7. Louis Vax, op. cit., p. 234.

8. Ibid., p. 46. 
glisser au bord de l'abîme, juste pour avoir la sensation d'être à la dérive, d'avoir perdu pour un temps les repères familiers et rassurants du monde quotidien ${ }^{9}$.

Cette rencontre d'irréconciliables implique la simulation d'un dérèglement régressif. Ce qui est ici côtoyé désigne une désagrégation psychique, et plus particulièrement une perte de contact normatif avec la réalité, plus souvent qu'autrement par l'effet d'une exacerbation du contact. La victime fantastique des motifs du surnaturel se voit alors touchée par divers symptômes: difficulté à lire la réalité, angoisse, perte ou intensification à outrance des sensations et sentiments, crainte d'instances supérieures, rupture de la raison discursive, renoncement à la rationalité, activité délirante, états d'allure démentielle, etc., tous symptômes de dissociation qui renvoient à des psychoses que l'on rassemble sous le terme de schizophrénie. Étymologiquement: fendre l'esprit. Irène Bessière dit justement du récit fantastique qu'il "se présente comme la transcription de l'expérience imaginaire des limites de la raison ${ }^{10}$.

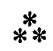

Dans cette perspective toute cailloisienne, l'intention générique conventionnée (entre auteurs et lecteurs) du fantastique serait de faire vaciller la raison. Et cela se réaliserait par introjection d'un hiatus dans la construction d'un monde hétérogène, construction référant elle-même à la convention d'un réel avéré (le principe clé, ici, étant celui de la convention, et non celui d'un réel avéré en sa pure extériorité). Je note d'ailleurs que je ne me permets que pour des raisons heuristiques le risque d'un glissement entre représentation - au sens d'une sensibilité au concret et réel avéré. Au sens strict, la littérature ne se rapporte à rien qui lui soit extérieur. "Les événements rapportés par un texte littéraire, écrit Todorov, sont des "événements" littéraires, et de mềme que les personnages, sont intérieurs au texte ${ }^{11}$. "Je ne reprends évidemment pas cette donnée archiconnue pour l'apprendre à qui que ce soit, mais pour nous remettre à l'esprit que ce qui est agi au cœur de la démarche fantastique ne renvoie pas en face à face un monde inventé et une supposée réalité, mais bien un hiatus et la convention de rationalité : en d'autres termes, ce qui est constitutif du fantastique, c'est qu'une brèche s'ouvre dans le principe de rationalité.

9. Rachel Bouvet, Étranges récits, êtranges lectures. Essai sur l'effet fantastique, Montréal, Balzac-Le Griot éditeur, coll. "L'univers des discours", 1998, p. 73.

10. Irène Bessière, Le récit fantastique. La poétique de l'incertain, Paris, Larousse université, coll. "Thèmes et textes", 1974, p. 62.

11. Tzvetan Todorov, Introduction à la littérature fantastique, Paris, Seuil, coll. "Poétique ", 1970, p. 64. 
Cette conception du fantastique, inspirée de Caillois et de Vax, permet de retenir deux postulats sous-jacents. Premier postulat: le fantastique repose sur une double transgression: non seulement une transgression du principe de rationalité, mais aussi une transgression du principe d'homogénéité (ou de non-contradiction); ce qui revient à dire que le fantastique est le genre de l'hétérogénéité. Or, ce concept d'hétérogénéité est pour moi fondamental. Je considère comme porteur de fantasticité potentielle tout procédé du programme textuel, représentations de lieux, de personnages, d'actions, toute scène proposant la mise en perspective d'une expérience de défaut de rationalité, toute entité, donc, dont l'apparition violerait les règles de l'homogénéité rationnelle. (Précisons du même souffle qu'un texte peut être porteur d'éléments potentiellement fantastiques (des motifs du surnaturel) sans être lui-même génériquement fantastique...)

Deuxième postulat: le texte fantastique implique une perception (un spectacle, comme dirait Charles Grivel ${ }^{12}$ ) ou un regard porté sur les choses et les êtres qui implique soit une fascination, soit une stupéfaction, et c'est dans la perspective d'un tel spectacle fascinant-stupéfiant que le texte fantastique fait reposer ses effets. Des effets qui se fondent, d'une part, sur une mise en rapport tensive du couple rationnel/surnaturel souvent d'ailleurs dans une montée paroxystique (il n'est pas rare, alors, qu'en amorce de cette démarche, la victime fantastique passe de regarder à voir, d'entendre à écouter, bref, d'une réception passive, banalisée, à une réception active, voire hyperactive) - et d'autre part, sur un mode de distribution de l'information (c'est-à-dire sur un faire informatif modalisé par les catégories véridictoires), un mode de distribution de l'information impliquant une perception ambiguë. Et c'est là, justement, que se dresse l'étrange contrat de véridiction que nous savons tous inhérent au genre fantastique, un contrat à la fois énoncif, c'est-à-dire qu'il s'inscrit dans le discours-énoncé et porte sur des valeurs pragmatiques, mais aussi un contrat énonciatif, lequel se manifeste par la structure même de l'énonciation, qui confine ici, nous l'avons dit, à l'ambiguité et, plus précisément, au spectacle de cette ambiguiité. Et voilà précisément ce à quoi il convient maintenant de nous intéresser...

Alors, que savons-nous de ce qui se passe dans le texte fantastique et qui puisse nous aider à comprendre les incidences du mode de

12. "N'est fantastique que ce qui s'offre au spectacle." Charles Grivel, "Horreur et terreur: philosophie du fantastique", Colloque de Cerisy. La littérature fantastique, Paris, Albin Michel, coll. "Cahiers de l'Herméneutique ", 1991, p. 174. 
distribution de l'information sur la fantasticité? Devant une question si large et si complexe, il nous faut évidemment choisir la retenue méthodologique. Nous aborderons donc une partie du texte, celle qu'il est convenu d'appeler l'incipit, au sens non restrictif de l'ouverture du texte fantastique, et peut-être un peu plus, car il semble particulièrement malaisé de délimiter le lever de rideau du texte fantastique. Je propose donc quelques remarques exploratoires et préliminaires sur cette caractéristique singulière du texte fantastique abordée plus haut et qui se condense en des formules, soit insinuantes de fantasticité, soit atténuantes, dans un mouvement alternatif et combinatoire de motifs du surnaturel et de motifs de la rationalité, de manière à contaminer les seconds par les premiers. Et plus précisément, je m'intéresserai, dans le sens de ce que j'ai dit jusqu'ici, au mode de décrochage, aux hiatus auxquels le fantastique impose d'assister dans les premiers pas du texte. À ce que Charles Grivel considère comme des failles ${ }^{13}$.

Je prendrai comme exemple les premières pages d'une nouvelle de Marie José Thériault, intitulée "Les cyclopes du jardin public ${ }^{14}$ ", une nouvelle fantastique de facture canonique ${ }^{15}$.

Commençons par un constat simple. Cette nouvelle de moins de dix pages fait environ 310 lignes. Une douzaine de lignes à peine sont consacrées à la scène morbide ultime et un peu plus de quatre de celles-ci suggèrent l'anthropophagie, qui d'ailleurs ne sera pas donnée en spectacle au lecteur. Cette scène finale à deux paliers n'occupe donc que $5 \%$ du texte. Les autres $95 \%$ ont donc pour fonction de préparer à l'étrangeté et au fantasme archétypal de la dévoration, c'est-à-dire d'opérer un embrayage fantastique.

J'entends l'expression "embrayage fantastique" au sens d'un embrayage générique, qui, par des critères de nature sociolectale, met en évidence l'axiologie sous-jacente au fantastique et signale au lecteur le mode de lecture qu'il convient d'appliquer. Pour tenir les exemples dans un registre simple, voyons comment sont touchés certains réseaux sémiotiques incontournables.

D'abord un cas d'embrayage temporel. Extrait 1: "La journée se déroulait à reculons avec une inquiétante lenteur. " $(C, 30)$ Ensuite un cas

13. Loc. cit., p. 171.

14. Marie José Thériault, "Les cyclopes du jardin public", La cérémonie, Montréal, La Presse, 1978, 139 p.

15. Je dis ce fantastique canonique au sens où "Les cyclopes du jardin public" répond aux normes courantes du genre - comme s'il en existait un modèle concret. Cela n'atténue en rien le caractère postmoderne de cette nouvelle, dont Sophie Wampach étudie un aspect caractéristique. Voir "L'inscription du fantastique dans "Les cyclopes du jardin public" de Marie José Thériault ", Maurice Émond (dir.), Les voies du fantastique québécois, Québec, Nuit blanche éditeur/CRELIQ, coll, "Séminairen, nº 3, 1990, p. 223-241. Désormais, les références à cette nouvelle seront indiquées par le sigle $C$, suivi du folio, et placées entre parenthèses dans le tèxte. 
d'embrayage spatial. Extrait $2:$ "[..] tout avait cette immobilité photographique des objets enfermés dans un ouf de verre." $(C, 30)$ Puis un cas d'embrayage actantiel. (À noter que, dans ces cas d'embrayage actantiel, le personnage est touché dans ses modalités, c'est-à-dire dans son vouloir-faire, dans son savoir-faire, dans son pouvoir-faire...) Extrait 3: "Bizarrement, il ne s'étonnait pas que tout parût ainsi pétrifié. Il ne remarquait pas davantage l'épaisseur du silence [...]" $(C, 30)$

À première vue, ces exemples sans doute parce que cette nouvelle est réputée appartenir au genre fantastique ${ }^{16}$, semblent marquer l'entrée de la narration dans un champ d'étrangeté propre au genre fantastique. Or, cela est-il si certain? Ne pourrait-on considérer, à la relecture de ces mêmes extraits, que les perceptions d'une journée se déroulant à reculons, d'une immobilité photographique des objets ou d'une épaisseur du silence puissent renvoyer à un procédé de métaphorisation - ce qui suggérerait une procédure discursive qui reposerait sur un jeu d'équivalence sémantique? Ces expressions, alors, apporteraient certes une coloration particulière, suggérant une perception assez schizoïde du monde, mais ne détonneraient pas dans un texte réaliste.

La métaphore, chacun le sait, est une figure de substitution qui, procédant par synecdoque, modifie le sens des mots. Or, l'embrayage fantastique ne peut se réaliser que lorsque et parce que le texte parvient, au contraire, à imposer son sens littéral. "Le récit fantastique est thétique, écrit Irène Bessière; il pose la réalité de ce qu'il représente ${ }^{17}$. "En d'autres termes, tant et aussi longtemps qu'il y a soupçon de métaphorisation, ces extraits ne peuvent poser leur représentation comme un réalisme second. J'emprunte l'expression "réalisme" second à Irène Bessière, qui précise: "Le projet littéraire fantastique est donc par nature antinomique: il doit allier son irréalité première à un réalisme second ${ }^{18}$. " Que ce réalisme second demeure faux dans son rapport à l'expérience humaine générale et à la motivation réaliste ne pose aucun problème, si du moins cette réalité parvient à constituer une hypothèse génériquement vraisemblable, c'est-à-dire fantastiquement vraisemblable. C'est là tout le jeu, l'ambiguïté et le mode de séduction de l'étrangeté fantastique.

16. Le péritexte est clair à ce sujet: la couverture de La cérémonie porte la mention "Contes", une inscription générique traditionnellement associée au fantastique; la quatrième de couverture renchérit, qui fait référence à des "contes bizarres" et à "un univers singulier, énigmatique [...] hors des frontières du temps et de l'espace». Par ailleurs, l'épitexte public et la réception critique (voir par exemple l'article de Michel Lord dans le Dictionnaire des ouvres littêraires du Québec, tome 6: 1976-1980: " ouvre marquante dans l'évolution du genre fantastique québécois") confirment cette perception de La cérémonie comme recueil de contes fantastiques, et, par glissement métonymique, de même pour "Les cyclopes du jardin public".

17. Irène Bessière, op. cit., p. 36.

18. Ibid., p. 32. 
Par ailleurs, nous savons que le principe d'embrayage de fantasticité exige une expulsion hors du présupposé de mimesis, de "quasi-chose", dit Ricour ${ }^{19}$, donc une opération de débrayage ${ }^{20}$ préalable du référent de réel avéré. Or, dans ces premiers exemples que nous venons de lire, s'il ne se dessine pas à proprement parler d'embrayage fantastique, on peut cependant dire que s'y met en marche, bien que sur un mode encore mineur, comme si une potentialité s'y dessinait, un débrayage du référent empirique, qui est alors entaché, si ce n'est de motifs du surnaturel, à tout le moins de motifs de l'étrangeté. Une incertitude, constitutive du genre fantastique, s'amorce par le fait de cette étrangeté potentielle. Une étrangeté qui n'est pas en elle-même proprement fantastique, mais qui contribuera, je dirais par accumulation et par connaissance générique, à induire la démarche fantastique (je dis "par connaissance générique", parce que je crois, avec Bakhtine et d'autres, que le genre est lui-même langage ${ }^{21}$ ).

Je rappelle ce que chacun sait, que le dispositif fantastique prescrit l'établissement de la valeur de référence (par description ou par présupposé de motivation réaliste, peu importe), puis un brouillage, et enfin la soumission à l'irrésolution. Ce que j'ai décrit plus haut participe de l'amorce du brouillage des certitudes.

Prenons un autre exemple d'embrayage spatial. Extrait 4: "[...] cette métropole vers laquelle il se dirigeait mais qui, il le constata soudain, semblait s'éloigner quand, au contraire, elle aurait dû se rapprocher" $(C, 31)$. Ici, la métaphorisation semble moins probable. Le défaut de perception est attribué au personnage en voie de devenir victime fantastique. Il n'y a pas encore l'assurance d'un motif surnaturel agissant, mais à tout le moins embrayage (consolidation) d'étrangeté.

À la fois par accumulation d'embrayeurs et peut-être par progression du défaut de perception, nous passons en effet de constats ambigus (les trois premiers exemples), assumés par une focalisation externe et omnisciente, au dévoilement d'un défaut de perception attribué au personnage. La rupture de logique conventionnelle est ainsi de plus en plus insinuée par différents types de perte de rapport à la réalité décrite. Le référent de

19. Paul Ricoeur, Temps et récit, 1, Paris, Seuil, 1983, p. 76.

20. A. J. Greimas et J. Courtés, se penchant sur le concept de shifter (introduit par Jakobson) en référence à l'énonciation, distinguent la procédure de débrayage (disengagement), soit une expulsion hors de l'instance d'énonciation, de la procédure d'embrayage (engagement), soit un effet de retour à l'énonciation. A.J. Greimas et J. Courtés, Sémiotique. Dictionnaire raisonné de la théorie du langage, tome 1, Paris Hachette, 1979, p. 349 (shifter), p. $79-82$ (débrayage) et p. 119-121 (embrayage).

21. Voir André Carpentier, "Aspects des genres littéraires appliqués à la science-fiction ", Aurélien Boivin, Maurice Émond et Michel Lord (dir.), Les ailleurs imaginaires. Les rapports entre le fantastique et la science-fiction, Québec, Nuit blanche éditeur, coll. "Les Cahiers du CRELIQ", 1993, p. 15-37. 
réalité objective est de plus en plus perturbé. Ce qui produit cette irrésolution, c'est que l'univers décrit ne répond à aucun système durable. Le fantastique se réalise dans l'instabilité, justement par débrayages / embrayages. C'est ainsi que travaillent les premières pages des "Cyclopes du jardin public". Et si l'on va plus loin dans le texte, jusqu'à considérer, par procédé heuristique, les premiers $95 \%$ comme incipit (façon d'outrer les concepts pour en vérifier la valeur), on constate que ces débrayages / embrayages se multiplient, s'agglomèrent, jusqu'à produire ce réalisme second dont parlait Irène Bessière et qui est fantastiquement vraisemblable.

\section{**}

En complément de ce qui vient d'être évoqué, voici une autre chose qui paraît d'une importance capitale et que j'appellerai la modalisation fantastique - en distordant un peu ce concept linguistique qui, je le rappelle, renvoie à un procédé par lequel un ensemble de marques formelles expriment l'adhésion d'un sujet d'énonciation au contenu de l'énoncé. Je parlerai de modalisation fantastique comme mode d'adhésion du texte au genre et en particulier à une poétique de l'incertitude. Je fais référence ici à ce que je décrivais, en ouverture, comme une stratégie du fantastique qui multiplie les formules, soit insinuantes (nous venons d'en voir brièvement le travail), soit atténuantes de fantasticité (c'est ce que nous verrons), dans un mouvement oscillatoire entre motifs de rationalité et motifs du surnaturel. C'est que les embrayeurs de fantasticité alternent, s'emmêlent à des formules qui tendent à maintenir un fond de référent empirique. Au regard des vingt-huit premières lignes des "Cyclopes du jardin public ", c'est-à-dire jusqu'à l'extrait $4(C, 31)$, qui me semble marquer la fin de cet incipit à valeur d'incertitude, on notera en effet des énoncés propres à maintenir le texte dans une motivation réaliste.

Voici deux exemples où la perception est (re)normalisée. Dans un cas, par un énoncé à portée générale: extrait 5 : "c'était là une nuit normale, fluide et si limpide qu'on pouvait s'y mouvoir librement." (C 30) Dans l'autre cas, par un énoncé qui associe le personnage à la perception normalisante: extrait 6 : "De l'endroit où il se trouvait, il distinguait nettement la silhouette bleue de la ville [...]. " $(C, 31)$

Ce que j'appelle la modalisation fantastique, qui détermine le type de fantasticité, et qui ouvre ici à une poétique de l'incertitude, œuvre donc, à l'ouverture des "Cyclopes du jardin public", par procédé d'alternance entre des énoncés porteurs et embrayeurs de fantasticité et des énoncés porteurs et embrayeurs de motivation réaliste. Car dans un fantastique comme celui-ci, les motifs du surnaturel font irruption dans le continuum de rationalité de manière progressive et dans un mouvement alternatif 
propre à créer l'incertitude. Ainsi le scandale de la déraison - qui s'installe clairement à compter du lieu de l'extrait $5(C, 30)$ - repose-t-il, tel que je l'ai mentionné plus haut, sur un incipit présentant des motifs dont la finalité diverge, tendant alternativement à insinuer un réalisme de référence et des ruptures de raison, de façon à produire un dérèglement régressif qui scandalise la raison.

Comme l'écrit Michel Lord:

[...] il existe un système de croyance et d'incroyance [...] formalisé esthétiquement dans et par le système narratif (forme du contenu) dans le texte fantastique et ce (double) système explique et structure le texte fantastique en tant précisément que texte fantastique de par le questionnement qu'il instaure - qui s'instaure - dans le récit autour de la question de la croyance ou de l'incroyance dans le phénomène étrange. Cet ancrage de contenu sert de déclencheur de la fantasticité. En d'autres termes, l'esthétique du questionnement sur l'étrange forme la matière / manière du récit fantastique. Ce serait là la pierre angulaire de la poétique du récit fantastique ${ }^{22}$.

Bien sûr, tous les textes fantastiques ne procèdent pas à la manière de celui-ci précisément. Il semble cependant que, dans nombre de textes fantastiques, ce procédé de double débrayage / embrayage et cette modalisation exercent en creux leur façon agissante, car le fantastique n'est pas, comme le croit Todorov, un lieu de partage, mais une scène de confrontation, qui postule le réel pour mieux le corrompre. Cette motivation traduit, nous ne l'ignorons pas, une lutte contre la banalisation du réel.

22. Michel Lord, op. cit., p. 42. 